\title{
Refractive correction with multifocal intraocular lenses after radial keratotomy
}

\author{
Bárbara Martín-Escuer ${ }^{1}$ - José F. Alfonso ${ }^{1,2}$ - Luis Fernández-Vega-Cueto $\mathbb{D}^{1} \cdot$ Alberto Domíngez-Vicent $^{3}$. \\ Robert Montés-Micó ${ }^{3}$
}

Received: 28 August 2018 / Revised: 10 December 2018 / Accepted: 19 December 2018 / Published online: 15 February 2019

(c) The Royal College of Ophthalmologists 2019

\begin{abstract}
Purpose To assess visual and refractive results of multifocal intraocular lens (IOLs) implantation for refractive correction after radial keratotomy (RK).

Methods In a retrospective non-comparative interventional case series, we analyzed the outcomes of multifocal IOL implantation performed in the context of cataract or refractive lens exchange surgery following RK. A total of 17 eyes from nine patients were included in the study. IOL power calculation was done using the Double-K formula. Refractive error was used to assess predictability, and distance-corrected visual acuity (DCVA) and uncorrected distance visual acuity (UDVA) values were used to assess the surgical procedure's efficacy and safety. Distance-corrected near visual acuity (DCNVA) was also determined.

Results Phacoemulsification and multifocal IOL implantation was successful in all cases, with neither complications nor adverse events. At 6 months postoperatively, monocular UDVA, DCVA, and DCNVA were $0.51 \pm 0.39,0.20 \pm 0.30$, and $0.11 \pm 0.11$, respectively (logMAR scale). More specifically, 35.29\% of the eyes had DCVA $\geq 20 / 20$ and $52.94 \%$ showed DCVA $\geq 20 / 25$. Regarding pre- vs. post-operative changes, $52.94 \%$ had lost one line of DCVA, $23.53 \%$ showed no changes, $11.76 \%$ had gained one line of DCVA, 5.88\% had gained two lines, and 5.88\% had gained three or more lines. The efficacy and safety indexes were 0.56 and 0.98 , respectively. As for near vision surgical outcomes, $29.41 \%$ of the eyes had DCNVA $\geq 20 / 20$ and $64.71 \%$ had DCNVA $\geq 20 / 25$. As for surgical accuracy, $29 \%$ of the eyes were within $\pm 0.50 \mathrm{D}$ of the target refraction, whereas $65 \%$ were within $\pm 1.00 \mathrm{D}$.

Conclusions Multifocal IOL implantation following radial keratotomy (RK) does not result in good distance visual performance, at least after 6 months of follow-up. Thus, this surgical approach has to be considered with only limited expectations.
\end{abstract}

\section{Introduction}

Radial keratotomy (RK), introduced by Fyodorov and Durnev [1], was in the past one of the most important refractive surgery techniques for myopia correction. The prospective evaluation of radial keratotomy (PERK) group

José F. Alfonso

j.alfonso@fernandez-vega.com

Fernández-Vega Ophthalmological Institute, Oviedo, Spain

2 Surgery Department, School of Medicine, University of Oviedo, Oviedo, Spain

3 Optics and Optometry and Vision Sciences Department, Faculty of Physics, University of Valencia, Valencia, Spain conducted an assessment of this technique prospectively in 1994 [2]. This study followed up on 693 eyes to evaluate the long-term (10 years) safety and efficacy outcomes of this technique and concluded that RK eliminates the need for distance optical correction in $70 \%$ of the patients with a reasonable level of safety, but they also reported a hyperopic shift of the refractive error, being progressive [2]. RK used declined with the advent of excimer laser-based techniques [3].

We are now seeing patients who have undergone RK and are now presenting with cataract or want to become spectacle-free again. Intraocular len (IOL) power calculation has been one of the main challenges that we have faced with these patients due to the above-mentioned hyperopic shift, and the difficult determination of the effective lens position and true corneal curvature $[4,5]$. Ma et al. [6] 
studied the accuracy of several IOL formulas in RK eyes and concluded that IOL power calculations require further enhancements when it comes to this type of patients. A recent study by Zhang et al. [7] encompassing 30 eyes and a 3 -year follow-up period concluded that phacoemulsification and IOL implantation with clear corneal incision were associated with good surgical outcomes.

Despite the intrinsic difficulties of IOL power calculations in these patients (mainly in presbyopia correction surgery) and the resulting impact upon visual and refractive surgical outcomes, several case reports focusing on presbyopia correction have been published [8-10]. Gupta et al. [8] reported the outcomes for two eyes implanted with hybrid monovision AcrySof IQ ReSTOR IOLs, concluding that their approach was an excellent option for patients aiming to be cataract and spectacle free.

Kim et al. [9] concluded, after refractive lens exchange with bilateral implantation of a rotationally asymmetric refractive multifocal IOL in two eyes from two unilateral RK patients, that this approach may benefit presbyopic patients. Moreover, Nuzzi et al. [10] also showed that the implantation of a customized toric multifocal IOL leads to good visual outcomes without regressions. All three case reports (Gupta's, Kim's, and Nuzzi's) concluded that multifocal IOL implantation might be a feasible approach to achieve presbyopia correction in RK patients. These studies are promising indeed, but larger samples are required for a thorough procedure evaluation.

In this context, the purpose of the present study was to report visual and refractive outcomes in a larger sample of RK patients who underwent cataract or refractive lens exchange surgery involving multifocal IOL implantation.

\section{Methods}

We retrospectively examined 17 eyes from nine consecutive patients who underwent cataract or refractive lens exchange surgery involving multifocal IOL implantation and who had also previously undergone RK surgery at the FernándezVega Ophthalmological Institute (Oviedo, Spain) between November 2004 and July 2016. In this most recent surgical procedure, we aimed to correct both refractive error and presbyopia in all patients. Informed consent was obtained from all patients once the nature and possible consequences of the study were explained to them.

\section{Pre-operative assessment}

Before IOL implantation, patients underwent a comprehensive ophthalmologic examination. The examination included uncorrected distance visual acuity (UDVA), distance-corrected visual acuity (DCVA) and refraction, slit-lamp examination, corneal topography, anterior chamber depth measurement and pachymetry (Orbscan II; Bausch \& Lomb, Rochester, NY), ocular biometry (Ocuscan; Alcon, USA and IOLMaster 500/700; Carl Zeiss Meditec, Jena, Germany), and indirect ophthalmoscopy.

\section{Surgical technique}

Surgery was carried out under peribulbar anesthesia. The main incision was performed on clear cornea provided that the RK radii were at least $3.2 \mathrm{~mm}$ apart, so that enough space was left to make the incision and to modulate its size, depending on the amount of astigmatism that was to be corrected. Alternatively, scleral access was used when corneal entry was not feasible. Capsulorhexis (under cohesive viscoelastic) and phacoemulsification (assisted by dispersive viscoelastic) were then performed. Prior to lens implantation, a capsular tension ring was inserted in all cases. To finish the surgery, an intracameral miotic solution (acetylcholine) was injected. For infection prophylaxis, 5\% and $10 \%$ iodine solution was applied onto the eyes and the skin, respectively, prior to the intervention, and $1 \mathrm{mg} / \mathrm{ml}$ of cefuroxime solution was injected into the anterior chamber once the surgical procedure was completed.

\section{IOLs power calculation and lenses used}

The double-K [11] formula was employed to calculate the power of the IOL to be implanted, based on the values yielded by the OcuScan and the IOLMaster 500/700. Preand post-RK keratometry values were also fed into this formula for IOL power calculation. In all cases, the target was fixed at $-1.00 \mathrm{D}$. Each eye included in this study had implanted one of the following seven multifocal IOL models: the Symfony ZXROO (Johnson \& Johnson, USA), the AcrySof ReSTOR SN6AD1 (Alcon, USA), the AcrySof ReSTOR SN60D3 (Alcon, USA), the Tecnis ZMBOO (Johnson \& Johnson, USA), the FineVision (Physiol SA, Belgium), the Acri.LISA 366D (Carl Zeiss Meditec, Germany), and the AT LISA tri 839MP (Carl Zeiss Meditec, Germany).

\section{Outcome indicators}

Pre-operative and post-operative visual acuity was evaluated and the occurrence of any adverse events or complications was recorded. The efficacy index (defined as the ratio between post-operative UDVA and pre-operative DCVA given in Snellen decimal notation) and the safety index (defined as the ratio between post-operative and preoperative DCVA) were also calculated. Snellen decimal visual acuity values were turned into $\log$ MAR values for statistical purposes. Distance-corrected near visual acuity 
(DCNVA) was also measured since one of the goals of this surgical approach was to provide spectacle-free functional near vision. All patients were followed up throughout a 6month period. For data analysis, we relied on Matlab software (MathWorks, Boston, USA). Normality was checked by means of the Kolmogorov-Smirnov test. A nonparametric Wilcoxon test was used to assess differences between post- and pre-operative outcomes. Differences were considered statistically significant when the corresponding $p$ value was smaller than 0.05 .

\section{Results}

Seventeen eyes of nine patients (six males and three females) were included in this study. All eyes had previously undergone RK and were at a later date implanted with a multifocal IOL. Mean patient age was 55.70 \pm 9.41 years and mean power of the implanted IOL was $23.68 \pm$ $6.92 \mathrm{D}$. This information together with other patient demographic data and pre- and 6-month post-operative eye examination outcomes are shown in Table 1. Considering that different multifocal IOL models were implanted, we created Table 2 so as to go into more detail and show individual values for each of the 17 eyes included in the study. Phacoemulsification and IOL implantation was performed successfully in all eyes and neither intra-operative complications occurred nor post-operative problems arose during the 6 months of follow-up.

\section{Efficacy and safety}

Mean monocular UDVA and DCVA were (logMAR notation) $0.51 \pm 0.39$ (about 20/63) and $0.20 \pm 0.30$ (about 20/ 32 ), respectively, at the 6-month follow-up. There were no statistically significant pre- vs. post-surgery changes neither for UDVA $(p=0.143)$, nor for DCVA $(p=0.58 \mathrm{P} 8)$. For near vision, DCNVA was (logMAR notation) $0.11 \pm 0.11$ (about 20/25).

Figure 1a shows cumulative frequency graphs for monocular UDVA (top) and DCVA (bottom). The percentage of eyes with better UDVA was higher before surgery (black bars) than after surgery (white bars); after surgery only $11.76 \%$ of the eyes (two) were $20 / 25$ or better and $35.29 \%$ of eyes (six) was $20 / 32$ or better. Figure 1c (at the bottom) is a cumulative frequency graph for pre- and postoperative DCVA. From this figure, we may observe that $35.29 \%$ of eyes (six) showed a DCVA of 20/20 or better before the surgery in contrast to the $5.88 \%$ of eyes (one) after the surgery. Same percentage $(52.94 \%$, nine eyes) was found for 20/25 or better both before and after the surgery. The resulting mean efficacy index was 0.56. Figure $1 \mathrm{~b}$ shows how UDVA and DCVA changed as a result of surgery. It is worth highlighting that 6 months after surgery, $52.94 \%$ (nine) of the eyes had lost one line of DCVA, $23.53 \%$ (four eyes) had stayed the same, $11.76 \%$ (two eyes) had gained one line of DCVA, $5.88 \%$ (one eye) had gained two lines, and $5.88 \%$ (one eye) had gained three or more lines of DCVA. The resulting mean safety index was 0.98 .
Table 1 Pre-operative and postoperative patient demographic data for our study sample

\begin{tabular}{lll}
\hline & Pre-operative values & 6 months postoperatively \\
\hline $\begin{array}{l}\text { Age (years) } \\
\text { logMAR visual acuity }\end{array}$ & $55.70 \pm 9.41(43$ to 69$)$ & \\
UDVA & $0.69 \pm 0.37(0.15$ to 1.30$)$ & $0.51 \pm 0.39(0.09$ to 1.30$)$ \\
CDVA & $0.18 \pm 0.26(0$ to 1.0$)$ & $0.20 \pm 0.30$ (0 to 1.30$)$ \\
CDNVA & N/A & $0.11 \pm 0.11(0$ to 0.30$)$ \\
Refraction (D) & & \\
M & $0.46 \pm 5.60(-15.00$ to +7.25$)$ & $-0.98 \pm 1.00(-3.12$ to +0.37$)$ \\
J0 & $0.01 \pm 0.58(-0.86$ to 1.47$)$ & $-0.15 \pm 0.35(-1.10$ to +0.31$)$ \\
J45 & $-0.04 \pm 0.18(-0.56$ to 0.26$)$ & $0.01 \pm 0.35(-0.82$ to +0.57$)$ \\
Keratometry (D) & & $37.01 \pm 3.23(31.50$ to 42.25$)$ \\
Steep & $37.05 \pm 3.28(32.00$ to 42.19$)$ & \\
Flat & $35.37 \pm 3.90(28.00$ to 41.75$)$ & \\
Axial length (mm) & $26.31 \pm 2.05(23.68$ to 30.89$)$ & \\
ACD (mm) & $2.83 \pm 0.18(2.54$ to 3.18$)$ & \\
IOL power (D) & $23.68 \pm 6.92(5$ to 33$)$ & \\
\hline
\end{tabular}

Results are given as mean \pm standard deviation values, with the corresponding range-minimum and maximum values-shown in brackets

UDVA uncorrected distance visual acuity, DCVA distance-corrected visual acuity, DCNVA distancecorrected near visual acuity, $M$ spherical equivalent, $J O$ Jackson cross-cylinder with the axes at 180 and 90 degrees, J45 Jackson cross-cylinder with the axes at 45 and 135 degrees, $A C D$ anterior chamber depth, N/A not available 


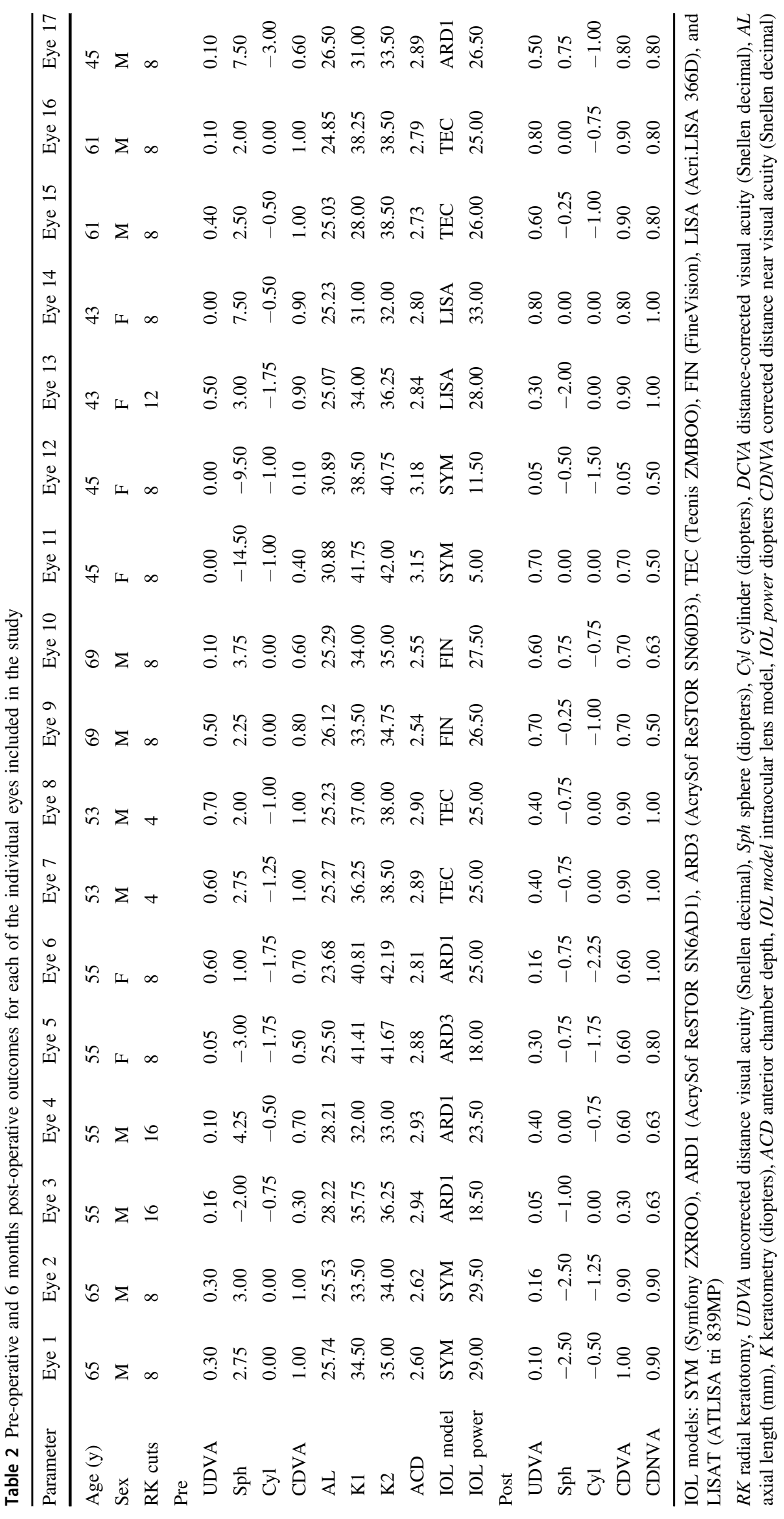



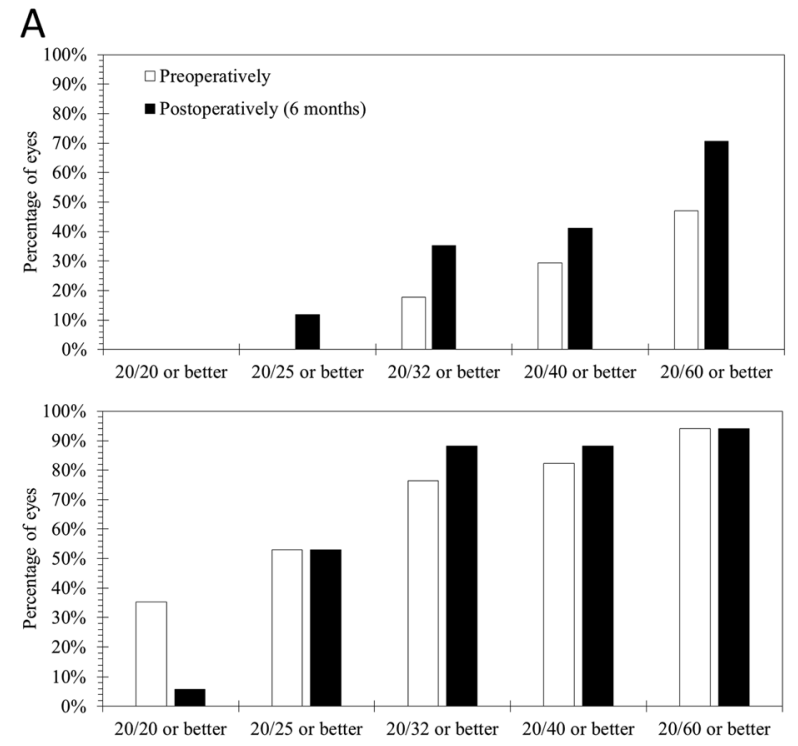

C

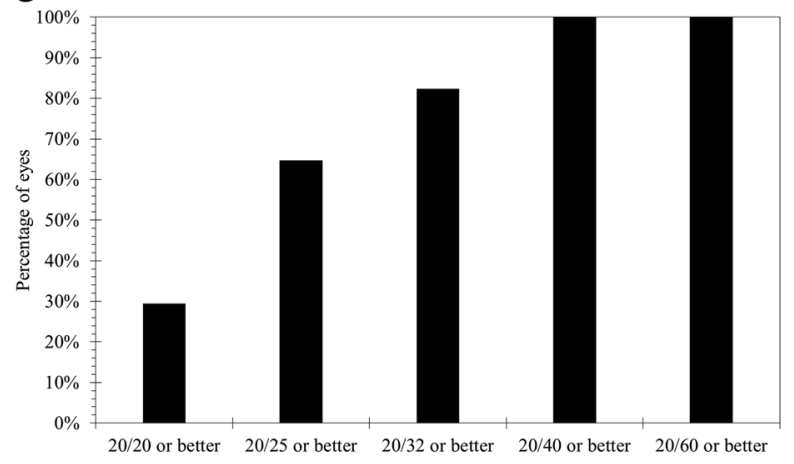

Fig. 1 a Cumulative proportion of eyes having a given outdistancecorrected visual acuity (UDVA, top) and best-distance-corrected visual acuity (DCVA, bottom) values, both preoperative and at 6 months postoperatively. b Post-operative changes in uncorrected distance visual acuity (UDVA) and best-distance-corrected visual acuity

Figure 1c shows the post-operative changes in DCNVA. At near, $29.41 \%$ of eyes (five) showed a DCNVA of 20/20 or better, $64.71 \%$ of eyes (11) of $20 / 25$ or better and $82.35 \%$ of eyes (14) of $20 / 32$ or better.

\section{Predictability}

Figure 1d is a scatter plot comparing for each individual patient achieved vs. attempted spherical equivalent (SE). The solid line represents the equality line (achieved refraction=attempted refraction), whereas the two dashed lines that run parallel to the solid one represent a $\pm 1.00 \mathrm{D}$ SE difference. When focusing on the SE component, $29 \%$ of eyes were within $\pm 0.50 \mathrm{D}$ of the target refraction, whereas $65 \%$ were within $\pm 1.00 \mathrm{D}$. Mean SE changed significantly, from $0.46 \pm 5.60 \mathrm{D}$ preoperatively to $-0.98 \pm$ $1.00 \mathrm{D}$ at 6 months $(p=0.008)$. Note that the SE before
B

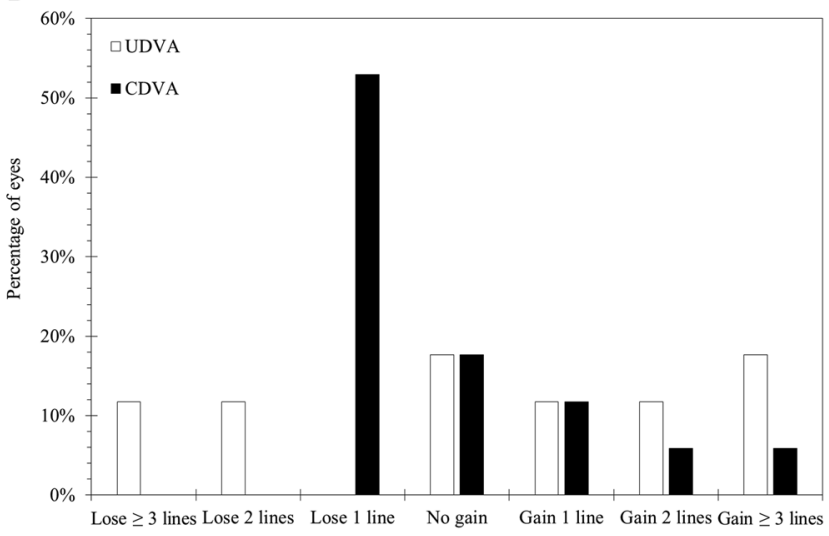

D

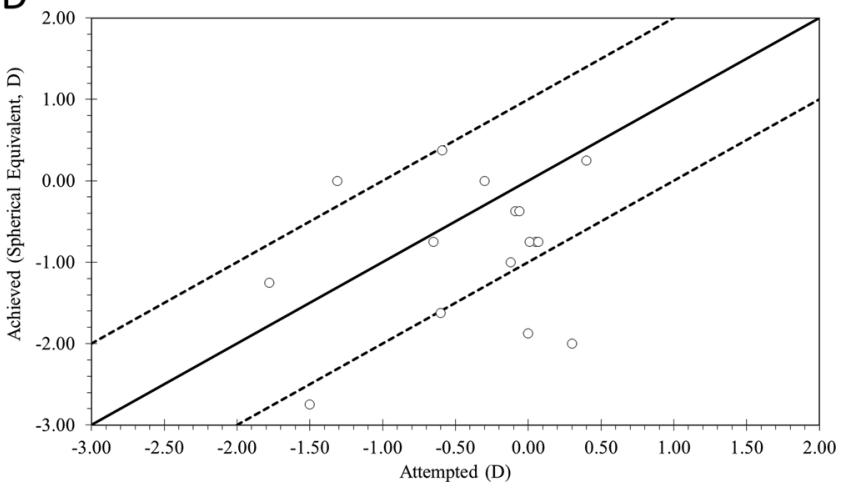

(DCVA) at 6 months postoperatively. c Cumulative proportion of eyes having a given best-distance-corrected near visual acuity (DCNVA) value at 6 months postoperatively. d Achieved versus attempted spherical equivalent at 6 months after the surgery (predictability)

surgery ranged from $-15.00 \mathrm{D}$ to $+7.25 \mathrm{D}$, and that interval had decreased at the last follow-up visit from -3.12 to +0.37 D. As for keratometry, no statistically significant changes were observed for flat and steep $K$ values $(p=$ 0.558 and $p=0.973$, respectively).

\section{Discussion}

Patients developing cataract need to be referred for cataract surgery with IOL implantation. The use of monofocal IOLs provides useful distance vision but fails to provide good unaided near vision. Monovision with monofocal IOLs or the implantation of multifocal IOLs are two alternative solutions that could therefore be considered. Patients who were referred to RK for refractive error correction with cataracts or clear lens with the desire to be spectacle free 
represent a difficult challenge to surgeons. The use of clear corneal incisions of different sizes in RK patients implanted with monofocal IOLs reported encouraging outcomes [7]. However, the use of monofocal IOLs in these patients only provide useful near vision based on a monovision strategy. Patients with multifocal IOLs were more likely to report spectacle independence that those that received monofocal implants for monovision [12]; namely, multifocal IOLs have proven to be effective at improving near vision [13]. Thus, in this context, the use of multifocal IOLs in patients who previously underwent RK is an option that has to be evaluated. Unfortunately, up to date, few cases have been reported but they have shown good and encouraging visual outcomes [8-10], so the purpose of our study was to further inspect this possibility.

The results obtained in our study at 6 months post surgery revealed no significant change compared before the surgery both in UDVA and DCVA (Table $1, p>0.1$ ). Mean values post surgery were about 20/63 and 20/32 for UDVA and DCVA, respectively. Only two eyes (11.76\%) achieved a UDVA of 20/25 or better (Fig. 1a, top). DCVA revealed similar values between pre- and post surgery but being more eyes before the surgery with a DCVA of 20/20 or better (6 eyes vs. 1 eye, Fig. 1a, bottom). This results in a low efficacy index (0.56).

Regarding DCVA (Fig. 1b), there were more eyes whose DCVA worsened (nine) after multifocal IOL implantation than eyes that stayed the same or gained one or more lines (eight). In this case, the safety index was better than the efficacy index (0.98).

A better outcome was found for DCNVA (about 20/25), which was equal to or better than $20 / 20$ for five eyes (Fig. 1c).

These results show that the procedure is safe, but it is not effective for distance vision enhancement. On the contrary, this technique provides good visual acuity for near vision, considering that the implanted IOL is a multifocal one. In relation to predictability, our results revealed that only a small percentage of eyes were within $\pm 0.50 \mathrm{D}$ of the target refraction (29\%), whereas 35\% of them (see Fig. 1d) ended up further than $1.00 \mathrm{D}$ away from the target refraction. The study population has been analyzed as a whole and mean values $( \pm \mathrm{SD})$ have been computed, but as reflected in Table 2 there are differences between the individual lens models. However, by looking at the individual data, it seems that all lens models perform similarly for this type of patients.

Previous studies by Gupta et al. [8], Kim et al. [9], and Nuzzi et al. [10] (see Table 3) involving the implantation of different multifocal IOL models reported better outcomes than ours. More specifically, Gupta et al. [8] used in two patients a hybrid monovision approach with the AcrySof IQ ReSTOR IOL and they were both able to achieve spectacle 
freedom. Monocular DCVA was 20/20 in three eyes and binocular DCNVA was 20/20 in both patients.

Kim et al. [9], with two unilateral RK patients referred for refractive lens exchange with the Oculentis IOL, reported 20/20 UDVA and J1 uncorrected near visual acuity (UNVA) for both patients. To conclude, Nuzzi et al. [10] implanted a custom multifocal toric IOL in an eye with previous cross-linking, resulting in monocular UDVA of 10/10 and UNVA of J1. Overall, in all three case reports visual outcomes were good without any adverse events. On the contrary, our results were not so good. We have to take into account that there are important differences between these studies; for example, different multifocal IOLs were used, hybrid monovision was used and patients with unilateral RK.

We also have to bear in mind for this type of patients two important factors: first, the fact that RK induces corneal irregularities (which leads to higher optical aberrations [14]), which, combined with complex optics (specially true for multifocal IOLs), may have an impact on visual function. Second, there is an overestimation of the corneal refractive power that is going to modify the correct power of the IOL (underestimate) to achieve emmetropia. Central corneal flattening (due to swelling and corneal edema) combined with an irregular cornea with radial incisions make it difficult to perform accurate keratometry measurement. In addition, diurnal variation or changes with time in this type of eyes may also have an impact upon final refractive and visual outcomes [15]. Taking repeated keratometry readings at different times is therefore essential. Moreover, if the cornea is not stable enough the surgeon may consider cross-linking so as to improve corneal stability. Elbaz et al. [16] did so in nine eyes showing that it is an effective method; however, the initial effect achieved at 6 months was then blunted by 12 months postoperatively. They concluded that longer follow-up is necessary to validate this possibility. As previously indicated, Nuzzi et al. [10] reported good outcomes in a patient implanted with a toric multifocal IOL who had previously undergone crosslinking.

It has been reported that RK patients showed a "multifocal lens effect" due to the power variation between the center and the periphery $[17,18]$. Power variation across the pupil may result in several images being formed onto the retina thus creating a multifocal effect and providing patients with pseudo accommodation. However, the combination of this corneal variability and multifocal effect with a multifocal design of the implanted IOL could deteriorate the quality of the retinal images and lead to a worsening of the visual function. Our results are in good agreement with this possibility.

One intrinsic limitation of our study is the use of different multifocal IOL models. It has been widely published that visual performance of multifocal IOL wearers depends on the IOL model and design that has been implanted: either bifocal, or trifocal or extended depth of focus IOLs, the magnitude of the near addition, etc.; all this may play a significant role in the quality of vision achieved by our patients.

In conclusion, our results do not support the use of multifocal IOLs in RK eyes. The low accuracy for IOL power calculation, plus the corneal irregularities lead us to consider for these patients alternative approaches, different from premium multifocal IOLs. Therefore, we believe that cataract and/or presbyopia correction in RK patients should be carried out with monofocal IOLs together with ophthalmic lenses for distance vision, if necessary, in residual reading glasses, or in bifocal or progressive lenses.

\section{Summary}

\section{What was known before}

- RK was one of the most popular refractive technique in the past.

- Intraocular power calculation is complicated in patients submitted to this technique.

\section{What this study adds}

- Our study shows the refractive results in RK patients who underwent a cataract surgery with a multifocal IOL implantation during 1-year follow-up.

- A pseudophakic multifocal IOL implantation is not a good treatment option in patients who have undergone RK and need a cataract surgery.

\section{Compliance with ethical standards}

Conflict of interest The authors declare that they have no conflict of interest.

Publisher's note: Springer Nature remains neutral with regard to jurisdictional claims in published maps and institutional affiliations.

\section{References}

1. Fyodorov SN, Durnev VV. Anterior keratotomy method application with the purpose of surgical correction of myopia. In: Fyodorov SN, editor. Pressing problems of ophthalmosurgery. Moscow: Moscow Research Institute of Ocular Microsurgery; 1977. p. 47-8.

2. Waring GO 3rd, Lynn MJ, McDonnell PJ, PERK Study Group. Results of the prospective evaluation of radial keratotomy (PERK) study 10 years after surgery. Arch Ophthalmol. 1994;112:1298-308. 
3. Duffey RJ, Leaming D. US trends in refractive surgery: 2002 ISRS survey. J Refract Surg. 2003;19:357-63.

4. Koch D, Liu J, Hyde L, Rock R, Emery J. Refractive complications of cataract surgery after radial keratotomy. Am J Ophthalmol. 1989;108:676-82.

5. Awwad S, Dwarakanathan S, Bowman R, Cavanagh H, Verity S, Mootha $\mathrm{V}$, et al. Intraocular lens power calculation after radial keratotomy: estimating the refractive corneal power. J Cataract Refract Surg. 2007;33:1045-50.

6. Ma JX, TM, Wang L, Weikert MP, Huang D, Koch DD. Comparison of newer IOL power calculation methods for eyes with previous radial keratotomy. Invest Ophthalmol Vis Sci. 2016;57:162-8.

7. Zhang J, Liu X, Wang J, Xiong Y, Li J, Li X, et al. Outcomes of phacoemulsification using different size of clear corneal incision in eyes with previous radial keratotomy. PLoS ONE. 2016;11: $\mathrm{e} 0165474$.

8. Gupta I, Oakey Z, Ahmed F, Ambati B. Spectacle independence after cataract extraction in post-radial keratotomy patients using hybrid monovision with ReSTOR $\left({ }^{\circledR}\right)$ multifocal and TECNIS $\left({ }^{\circledR}\right)$ monofocal intraocular lenses. Case Rep Ophthalmol. 2014;5: 157-61.

9. Kim K, Seok K, Kim W. Multifocal intraocular lens results in correcting presbyopia in eyes after radial keratotomy. Eye Contact Lens. 2017;43:e22-e25.

10. Nuzzi R, Monteu F, Tridico F. Implantation of a multifocal toric intraocular lens after radial keratotomy and cross-linking with hyperopia and astigmatism residues: a case report. Case Rep Ophthalmol. 2017;8:440-5.

11. Aramberri J. Intraocular lens power calculation after corneal refractive surgery: double-K method. J Cataract Refract Surg. 2003;29:2063-8

12. Wilkins MR, Allan BD, Rubin GS, Findl O, Hollick EJ, Bunce C, Study Group. et al. Randomized trial of multifocal intraocular lenses versus monovision after bilateral cataract surgery. Ophthalmology. 2013;120:2449-.e1.

13. de Silva SR, Evans JR, Kirthi V, Ziaei M, Leyland M. Multifocal versus monofocal intraocular lenses after cataract extraction. Cochrane Database Syst Rev. 2016;12:CD003169.

14. Applegate RA, Howland HC, Sharp RP, Cottingham AJ, Yee RW. Corneal aberrations and visual performance after radial keratotomy. J Refract Surg. 1998;14:397-407.

15. Kemp JR, Martinez CE, Klyce SD, Coorpender SJ, McDonald MB, Lucci L, et al. Diurnal fluctuations in corneal topography 10 years after radial keratotomy in the Prospective Evaluation of Radial Keratotomy Study. J Cataract Refract Surg. 1999;25:904-10.

16. Elbaz U, Yeung SN, Ziai S, Lichtinger AD, Zauberman NA, Goldich Y, et al. Collagen crosslinking after radial keratotomy. Cornea. 2014;33:131-6.

17. Maguire LJ, Bourne WM. A multifocal lens effect as a complication of radial keratotomy. Refract Corneal Surg. 1989;5:394-9.

18. Moreira H, Garbus JJ, Lee M, Fasano A, McDonnell PJ. Multifocal corneal topographic changes after radial keratotomy. Ophthalmic Surg. 1992;23:85-9. 